Case Report

\title{
Giant Parotid Pleomorphic Adenoma with Atypical Histological Presentation and Long-Term Recurrence-Free Follow-Up after Surgery: A Case Report and Review of the Literature
}

\author{
Mohammed AlKindi $\left(\mathbb{D},{ }^{1}\right.$ Sundar Ramalingam $\left(\mathbb{D},{ }^{1}\right.$ Lujain Abdulmajeed Hakeem (D), \\ and Manal A. AlSheddi $\mathbb{1}^{2}$ \\ ${ }^{1}$ Department of Oral and Maxillofacial Surgery, College of Dentistry, King Saud University, Riyadh, Saudi Arabia \\ ${ }^{2}$ Department of Basic Sciences, College of Dentistry, Princess Nourah Bint Abdulrahman University, Riyadh, Saudi Arabia \\ Correspondence should be addressed to Sundar Ramalingam; sundar.ksu@gmail.com
}

Received 5 July 2020; Revised 14 August 2020; Accepted 21 August 2020; Published 1 September 2020

Academic Editor: Pravinkumar G. Patil

Copyright (C) 2020 Mohammed AlKindi et al. This is an open access article distributed under the Creative Commons Attribution License, which permits unrestricted use, distribution, and reproduction in any medium, provided the original work is properly cited.

\begin{abstract}
Salivary gland tumors (SGT) comprise $3 \%$ of all head and neck tumors, are mostly benign, and arise frequently in the parotid gland. Pleomorphic adenoma (PA) is the commonest SGT, representing $60-70 \%$ of all benign parotid tumors. Clinically, parotid PA presents as irregular, lobulated, asymptomatic, slow-growing preauricular mass, involving both superficial and deep lobes, and could grow to gigantic proportions. Histologically, PA has epithelial and mesenchymal elements in chondromyxoid matrix and is managed surgically. Based on a review of 43 cases reported in English literature since 1995, giant parotid PA is reported as large as $35 \mathrm{~cm}$ (diameter) and $7.3 \mathrm{~kg}$ (resected weight). Although rare, 10 cases of malignant transformation were reported in the review. Surgical management included extracapsular dissection (ECD), superficial parotidectomy, and total parotidectomy for benign tumors, and adjuvant radiation or chemotherapy for malignant tumors. We further present the case of a 36-year-old healthy male with slow-growing and asymptomatic giant parotid PA, of 4-year duration. The patient presented with firm, lobulated preauricular swelling, provisionally diagnosed as PA based on radiographic and cytological findings. The tumor was resected through ECD, and the patient had uneventful postoperative recovery and a 7-year recurrence-free follow-up period. Histological examination revealed epimyoepithelial proliferation punctuated by chondromyxoid areas, with extensive squamous metaplasia and keratin cysts. To the best of knowledge from indexed literature, giant parotid PA is rarely reported in Saudi Arabia. In addition to its rarity, this case is reported for its benign nature despite atypical histological presentation, successful surgical management without complications, and long-term recurrence-free follow-up. Based on this report, clinicians must be aware of atypical histological presentations associated with PA and plan suitable surgical management and follow-up to avoid morbidity. Nevertheless, attempts must be made to diagnose and manage these lesions at an early stage and before they reach gigantic proportions.
\end{abstract}

\section{Introduction}

Neoplastic lesions of the salivary glands are uncommon and comprise less than $3 \%$ of all reported head and neck tumors $[1,2]$. Nearly $80 \%$ of the reported salivary gland tumors (SGT) are benign and occur predominantly in major salivary glands, with the parotid gland being the commonest site (70$80 \%)$ [2, 3]. Often presenting as slow-growing, painless masses, tumors involving major salivary glands are rarely aggressive or malignant $(<10 \%)$. On the contrary, tumors of minor salivary glands while occurring rarely have a preponderance to be malignant $(80-90 \%)[2,3]$. Pleomorphic adenoma (PA) is the commonest SGT, accounting for almost $60-80 \%$ of all benign SGT and $60-70 \%$ of all parotid gland tumors $[1,3]$. Clinically, PA presents as an irregular, rubbery, lobulated, slow-growing mass without any associated pain or discomfort. The presenting complaint is typically related to unpleasant or unesthetic facial appearance, which when 
disregarded can lead to patients reporting with huge lesions [4]. Reports in the literature suggest resected dimensions of $\mathrm{PA}$ to be frequently in the range of $2 \mathrm{~cm}$ to $6 \mathrm{~cm}$ and rarely reaching even up to $25-35 \mathrm{~cm}[4,5]$.

As the name suggests, PA is histologically categorized as a benign mixed (pleomorphic) tumor of ductal and myoepithelial cell origin. Owing to the pluripotential nature of myoepithelial cells, the tumor is composed of epithelial and fibrous, myxoid, and cartilaginous mesenchymal elements surrounded by a pseudocapsule, with occasional squamous metaplasia $[3,4,6]$. While the diagnosis of PA is based primarily on clinical and histological findings, the mainstay of management is by surgical excision $[1,4]$. Depending upon their size and depth of involvement, parotid PA is surgically managed either by superficial parotidectomy (SP), extracapsular dissection (ECD), or total parotidectomy (TP). All of the above procedures carry the risk of postoperative facial nerve paralysis and Frey's syndrome $[1,7]$. Recurrence is usually associated with inadequate clearance and incomplete removal of pseudocapsule, and malignant transformation has been reported with large, long-standing PA $[4,7]$.

Although uncommon, giant pleomorphic adenomas of the parotid gland have been reported. An electronic search of the English-language articles through the Medline, Scopus, and Google Scholar databases revealed 43 reports of giant parotid PA since 1995 [4, 8-32], with sizes ranging up to $28-33 \mathrm{~cm}[15,18,27]$ and tumor mass ranging up to $6.85-$ $7.3 \mathrm{~kg}[10,27]$. Major reasons for patients reporting with large PA are lack of resources, inaccessibility to medical facilities, fear of surgical procedure, and poor awareness, compounded by an asymptomatic and slow-growing lesion [4]. The aim of this paper is to add to the existing scientific literature, a case of giant parotid PA treated surgically by ECD, followed by uneventful postoperative recovery and a 7-year recurrence-free follow-up. This paper also attempts to highlight the benign nature of parotid PA, despite the atypical histological presentation, which could be associated with it.

\section{Case Report}

A 36-year-old healthy male patient reported to the oral and maxillofacial surgery outpatient clinic at the College of Dentistry and Dental University Hospital, King Saud University, in October 2012. The patient sought medical attention for a slow-growing, painless swelling in the right preauricular region. History revealed that the patient noticed the swelling almost 4 years ago, and since then, it had gradually increased in size with no obvious symptoms or changes to the overlying skin. Upon interviewing, the patient reported no relevant medical or surgical history and mentioned fear of surgery and absence of discomfort as reasons for delaying medical consultation, in spite of an unesthetic facial appearance.

Clinical examination revealed a firm, nontender, nodular, and mobile swelling with apparently normal overlying skin. The swelling extended superoinferiorly from the level of the external ear to the lower border of the mandible and anteroposteriorly from the angle of the mouth to the posterior border of the mandible. There was no lymph node involvement or facial nerve deficit (Figure 1). Preoperative computed tomography (CT), magnetic resonance imaging (MRI), and fine-needle aspiration cytology (FNAC) were ordered. CT with contrast revealed a well-defined mass lesion in the superficial lobe of the right parotid gland, without any underlying bony erosion and normal-appearing pharynx, larynx, and parapharyngeal spaces. While confirming the CT findings, head and neck MRI further demonstrated a well-demarcated, heterogeneous, mass lesion measuring $10 \times 7 \times 8 \mathrm{~cm}$ in maximum dimension (Figure 2 ). FNAC showed numerous scattered groups and clusters of plasmacytoid myoepithelial cells, associated with a chondromyxoid matrix. A provisional diagnosis of PA with no malignant tendency was arrived at based on CT, MRI, and FNAC findings.

Surgical removal of the right parotid SGT under general anesthesia was planned and explained to the patient. Following informed consent, the lesion was excised completely through ECD, with preservation of all branches of the facial nerve. The right parotid gland was approached using a cervically extended preauricular skin incision. A clearly discernible plane of dissection around the tumor was used for dissecting the tumor mass, without any iatrogenic damage to the facial nerve branches. Owing to the long-standing nature, multiple small feeder vessels had to be ligated circumferentially around the tumor to achieve hemostasis. The intraoperative period was unremarkable, and the patient did not require any blood transfusions (Figure 3 ). The resected mass was bilobed and ovoid in shape with a final dimension of $7 \times 13 \times 7 \mathrm{~cm}$ and weighing $1.2 \mathrm{~kg}$.

Histopathological examination of the excised specimen gave a gross appearance of a partially encapsulated mass containing myoepithelial and ductal proliferation. There was marked stromal hyalinization, squamous metaplasia, and keratinization. Some epithelial islands exhibited papillary configuration, along with large cysts and inflammation. Chondromyxoid changes and fibrosis were evident throughout the tumor. Certain foci of tumor islands were seen approaching and breaking through it. Hematoxylin and eosin (H\&E) stained sections revealed a partially encapsulated tumor with variable histopathological features and focal effacement of the fibrous capsule. The tumor typically showed epithelial/myoepithelial proliferation punctuated by chondromyxoid areas. Aggregates of plasmacytoid myoepithelial cells as well as ducal differentiation surrounded by clear myoepithelial cells were evident. Based on the above findings, a final diagnosis of benign PA was reached. While the tumor sections showed no evidence of malignant change, there was extensive squamous metaplasia and keratin cyst formation, which were atypical for PA (Figure 4). The patient was therefore advised close follow-up, once every month for the first year postoperatively and subsequently once in six months.

At 6 weeks postsurgery, the patient had unremarkable wound healing without any neurological deficit of the facial nerve (Figure 5). As of December 2019, the patient had a recurrence-free follow-up period of 7 years and presented with normal activity of muscles of facial expression, indicating the absence of any long-term facial nerve weakness (Figure 6). 


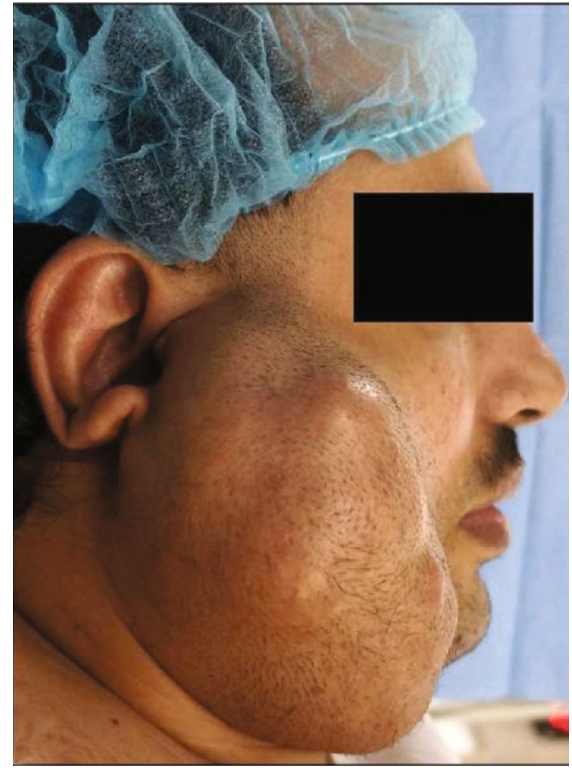

(a)

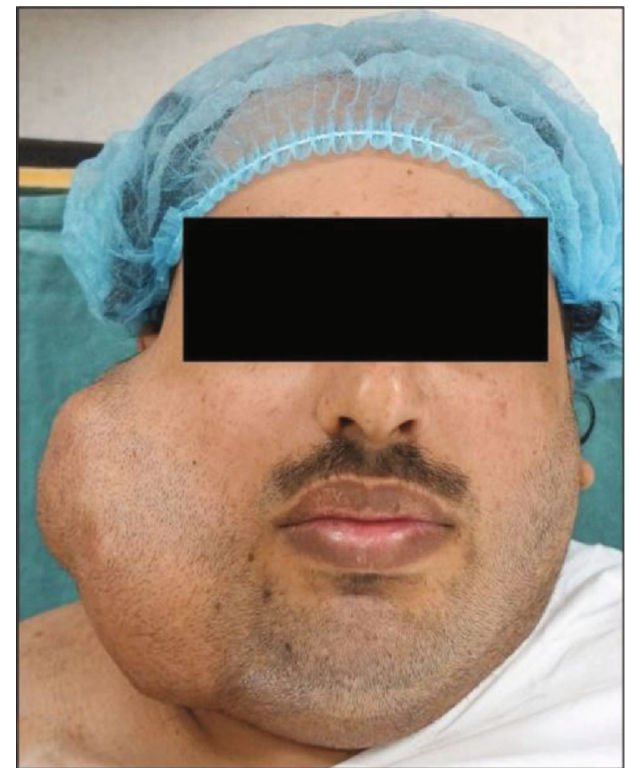

(b)

Figure 1: Preoperative clinical photograph of the right preauricular swelling. (a) Right lateral facial view shows the swelling extending superoinferiorly from a point anterior to the helix of the external ear until the lower border of the mandible; anteroposteriorly, the swelling is seen extending from the angle of the mouth to the posterior border of the mandible; the ear lobe is deflected outward and elevated, and the skin overlying the swelling appears free of any ulceration, puckering, or discharge. (b) Frontal facial view shows the swelling causing facial asymmetry and obliterating the view of most of the right external ear; there is no clinical evidence of facial nerve weakness or deficit.

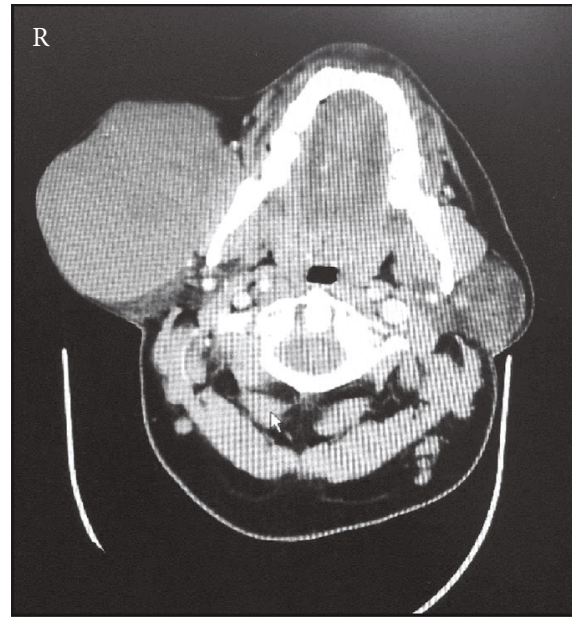

(a)

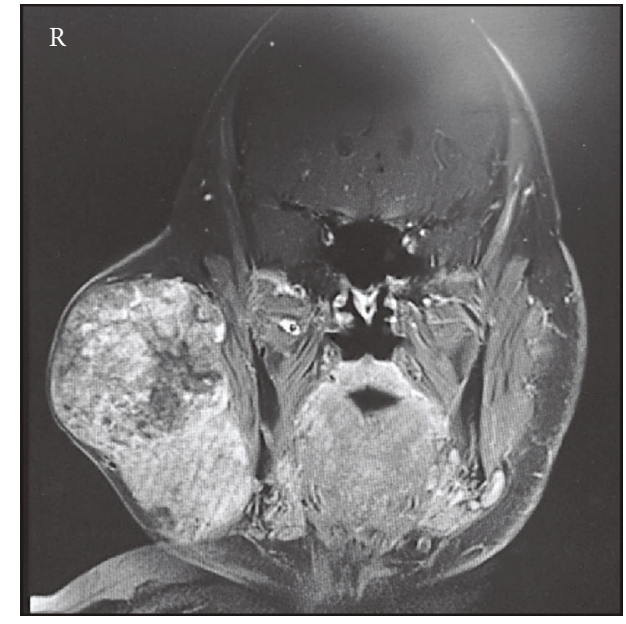

(b)

Figure 2: Preoperative radiographic examination of the right preauricular swelling. (a) Contrast-enhanced computed tomography axial section at the level of mandibular teeth shows a well-defined mass lesion in the superficial lobe of the right parotid gland, without any underlying bony erosion and normal-appearing pharynx, larynx, and parapharyngeal spaces. (b) Magnetic resonance imaging coronal section along the posterior border of the mandible shows a large, heterogeneous, well-demarcated solid mass lesion within the right parotid superficial lobe and measuring $10 \times 7 \times 8 \mathrm{~cm}$ at maximum dimensions.

\section{Discussion}

The parotid gland is the largest salivary gland with an average weight ranging from 0.015 to $0.021 \mathrm{~kg}$ and measuring approximately $5.8 \times 3.4 \mathrm{~cm}$ in the craniocaudal and ventrodorsal dimensions, respectively. Being the first salivary gland to develop in utero, during the $6^{\text {th }}$ gestational week, it is anatomically located bilaterally between the mastoid process of temporal bone and ramus of the mandible. The terminal branches of the facial nerve are an important anatomic landmark which divide the parotid gland into its superficial and deep lobes [33]. Although SGT are uncommon, they are 


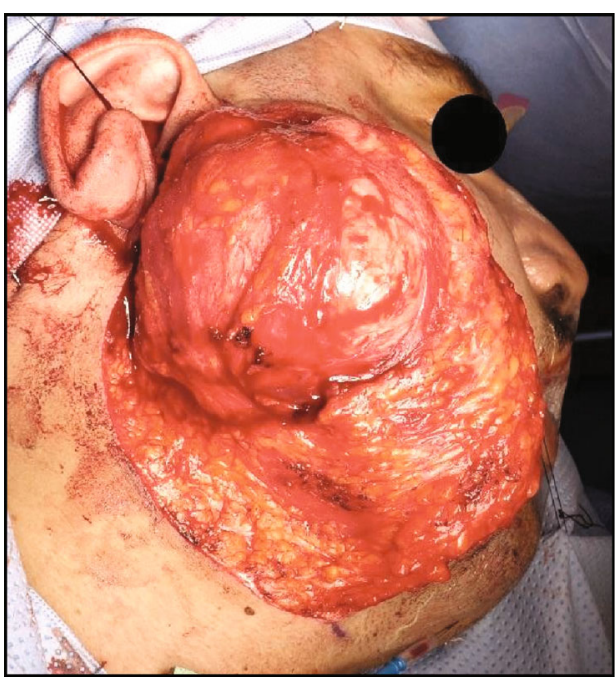

(a)

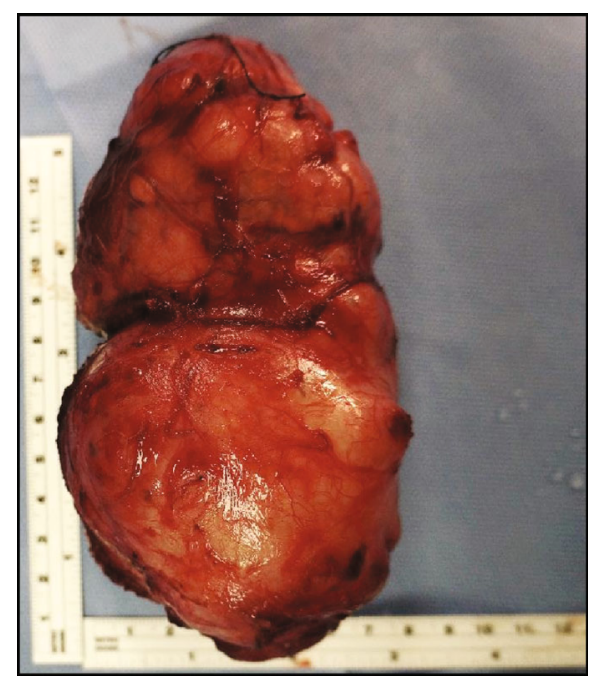

(b)

FIGURE 3: Intraoperative photograph showing (a) the surgical plane for extracapsular dissection of the right parotid tumor and (b) the excised tumor specimen.

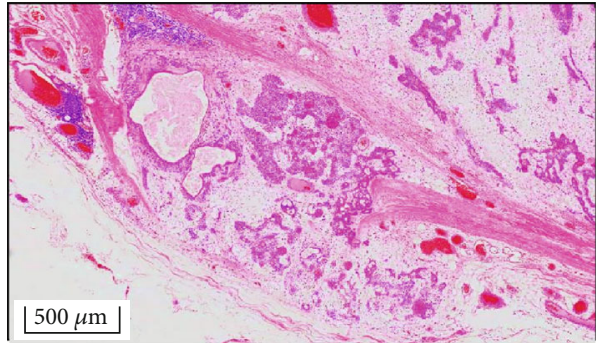

(a)

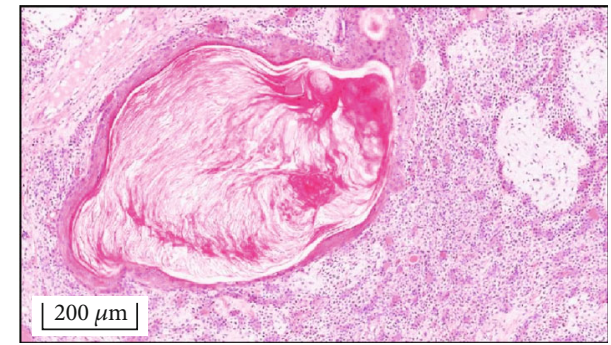

(b)

FIGURE 4: Histological examination of the excised tumor specimen showing (a) a partially encapsulated mass lesion containing myoepithelial and ductal proliferation, with stromal hyalinization, squamous metaplasia, and keratinization; epithelial islands exhibiting papillary configuration, large cysts surrounded by inflammation, focal areas of chondromyxoid changes and fibrosis, and tumor islands approaching and penetrating the capsule are evident (HE original magnification $\times 4$ ); (b) extensive squamous metaplasia and keratin cyst formation are conspicuous at higher magnification (HE original magnification $\times 10$ ).

predominantly benign and are reported frequently in the parotid gland [2]. The present report details a case of giant PA in the right parotid gland, along with its surgical management, histological presentation, and long-term recurrence-free follow-up.

Pleomorphic adenoma is the commonest mixed SGT arising in the parotid gland, and several cases have been reported in the literature. Although there are no specific physical criteria outlined in the literature to classify giant parotid PA, the earliest recorded case report dates back to 1863 [27]. In this report, Spence reported a mixed tumor involving lateral face and neck and a resected mass weighing greater than $1 \mathrm{~kg}$ [27]. Similarly, Short and Pullar (1956) reviewed and reported a case of giant parotid PA weighing about $2.3 \mathrm{~kg}$ [27]. In a report reviewing 31 cases of giant parotid PA over a period of 140 years by Schultz-Coulon, the resected tumor weights ranged from 1.0 to $26.50 \mathrm{~kg}$, with a greater female predilection (64.5\%) and only 3 cases of malignant transformation [15]. Based on a review of the 10 largest parotid PA published between 1863 and 1994, Buenting et al. reported resected tumors ranging in weight from 2.83 to $26.50 \mathrm{~kg}$, in patients with age ranging from 25 to 85 years and $90 \%$ female predilection [10].

A literature search was conducted to review giant parotid PA cases reported in Medline, Scopus, and Google Scholar databases. The search strategy involved a combination of search keywords including "GIANT", "PAROTID GLAND", and "PLEOMORPHIC ADENOMA", based on which 288 articles were identified from the three databases (Medline-66; Scopus-63; Google Scholar-159). Reviewing their abstracts, articles published in English only were selected based on them reporting a case or series of cases of giant parotid PA, including clinical, radiographic, histologi$\mathrm{cal}$, and surgical outcomes. Twenty-six articles published in English language were identified [4, 8-32] since 1995, and they reported 43 cases of PA in total. While most of the articles selected for review were single case reports, three articles were case series reporting about two cases [16], three cases 


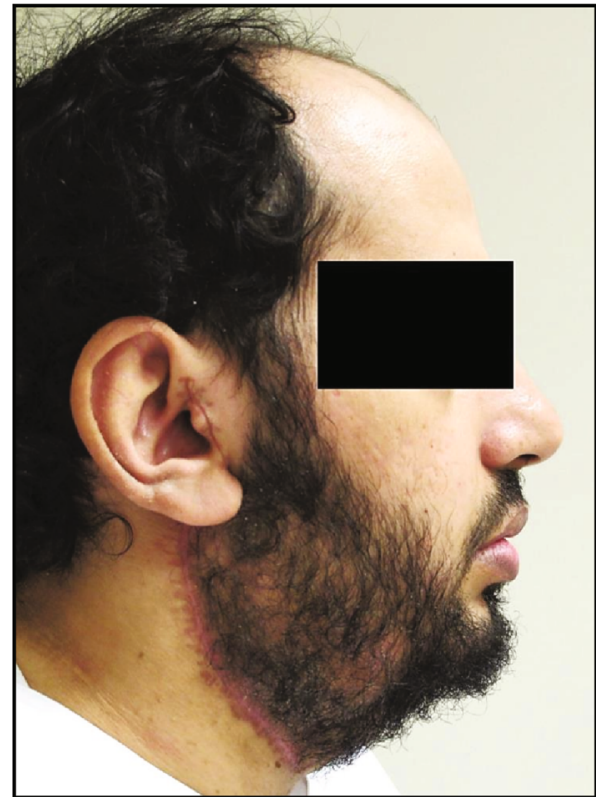

(a)

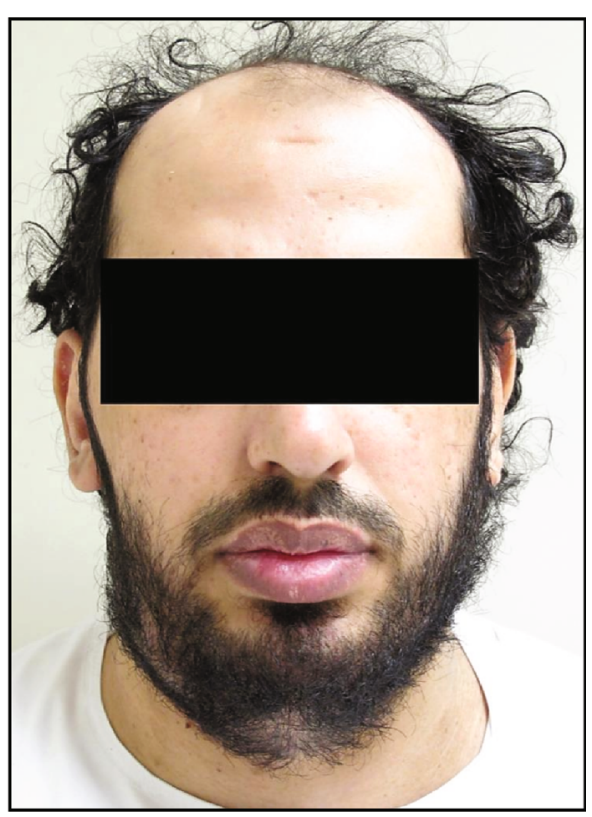

(b)

FIgURE 5: Postoperative clinical photograph taken 6 weeks postsurgery. (a) Lateral facial view shows healing surgical incision without any obvious postoperative sequelae. (b) Symmetric facial appearance observed in the frontal facial view, with no clinical weakness of muscles of facial expression.

[17], and 15 cases [32], respectively. The largest case series in the present review, comprising 15 PA patients, was reported by Pareek et al. [32].

Although majority of the reported cases were in patients aged 45 years or older $(n=30,69.8 \%)$, the age at clinical presentation and surgery ranged from 21 to 92 years. The asymptomatic, slow-growing nature of pleomorphic adenoma was evidenced by the fact that the duration from the first observation of lesion to reporting for treatment varied from 5 to 35 years. Demographically, there were more females $(n=25,58.1 \%)$ than males, and all reported cases were unilateral, with the right side $(n=23,53.5 \%)$ affected more than the left. In terms of clinical dimension, the tumors ranged from 3 to $5 \mathrm{~cm}$ in diameter $[16,17]$, until $35 \times 28 \mathrm{~cm}$ [27], in two perpendicular planes. The clinical dimensions corroborated with the resected tumor weight, wherein the smallest tumor weighed $0.12 \mathrm{~kg}$ [16] and the largest weighed $7.3 \mathrm{~kg}$ [27]. Predominant clinical presentation of the tumors was that of a large, lobulated, and pedunculated mass with apparently normal overlying skin. Ulceration of the skin was reported in eight patients, out of which five were reportedly associated with malignant change [15, 23, 24, 30, 32], and the remaining three were due to injury $[18,29,32]$. Anatomically, the tumor more commonly involved the superficial lobe of the parotid gland $(n=29,70.7 \%)$ giving rise to the clinical presentation of a large preauricular mass. Nevertheless, when the deep lobe of parotid and parapharyngeal spaces were involved by the tumor, patients presented with an intraoral swelling leading to soft palate displacement, difficulty in swallowing and breathing, and obstructive sleep apnea $[11,13,16,17,22,25]$. While diagnosis was primarily based on clinical and radiographic (USG, CT, and MRI) findings, preoperative diagnosis was established through FNAC in most cases. The clinical, radiographic, surgical, and histological findings in the reviewed case reports are detailed in Table 1.

Preoperative diagnosis of PA is routinely based on clinical findings, supplemented by radiological investigations such as CT, MRI, and USG [2]. The role of FNAC in arriving at a provisional diagnosis has been debated and considered nonrepresentative due to varying histological patterns at different sites within the same tumor [34]. In terms of histopathological diagnosis, the characteristic feature of PA is its histological diversity and differing arrangements of epithelial and mesenchymal tissue elements. Das and Anim [34], based on a study comparing FNAC and histological sections in PA, reported consistent findings of epithelial cells in a myxoid stroma through cytological and histological examination. Nevertheless, they reported better characterization of oncocytic changes such as acini, giant cell and mucus globule formation, and squamous and chondroid metaplasia in histological sections [34]. Preoperative diagnosis of PA in the present case was based on a combination of clinical examination, radiographic investigations, and FNAC. Our radiographic finding (CT and MRI) of well-demarcated, lobulated, and heterogeneous mass lesion involving the parotid gland and FNAC finding of plasmacytoid epithelial cells in a chondromyxoid matrix were in coherence with the majority of cases reported in the review (Table 1). Additionally, necrotic changes $[10,24,30]$ and calcifications $[4,30]$, within the tumor, have also been reported in the literature, based on CT and in association with malignant change $[24,30]$. The combination of CT and MRI enables optimum preoperative diagnosis of pleomorphic adenomas and precise planning of the surgical approach for tumor resection [11]. In addition to volumetric information, CT with contrast provides 


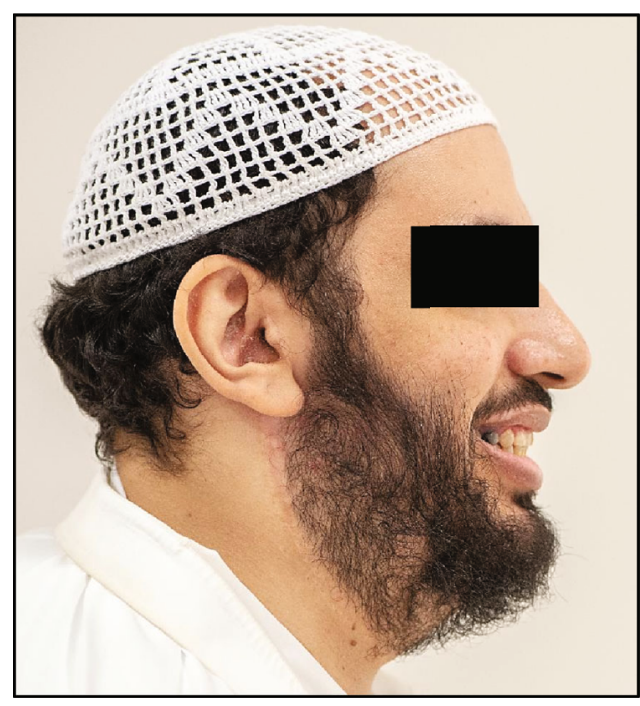

(a)

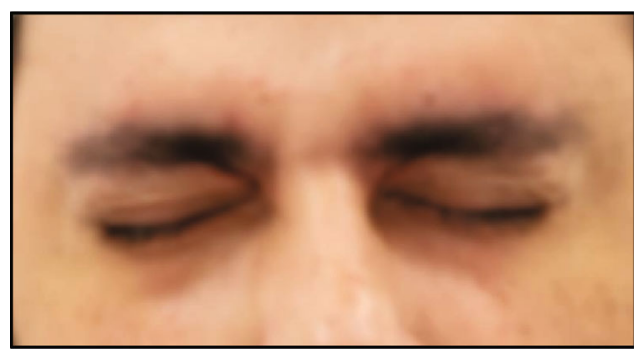

(c)

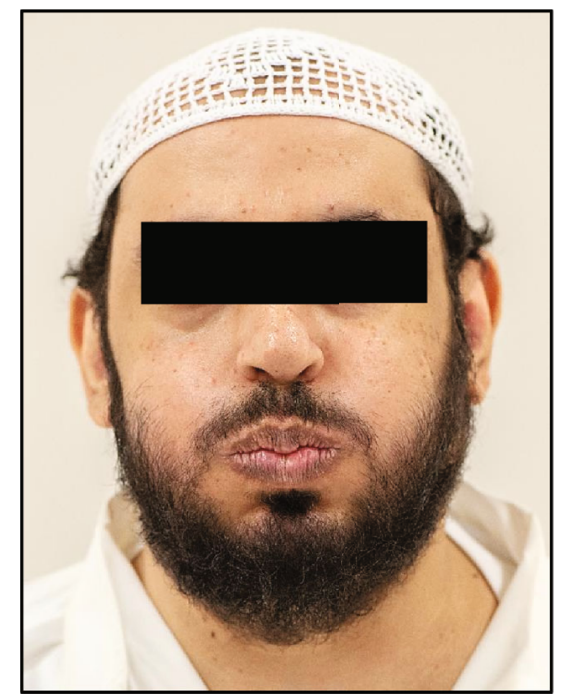

(b)

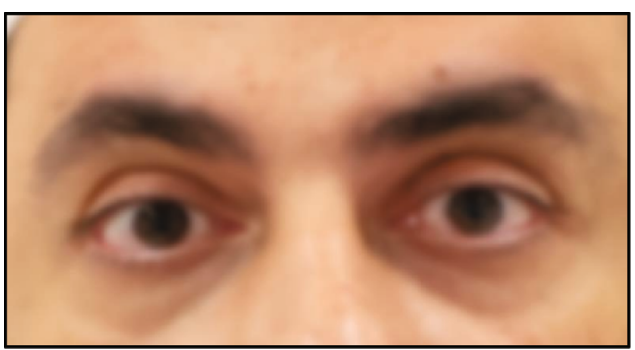

(d)

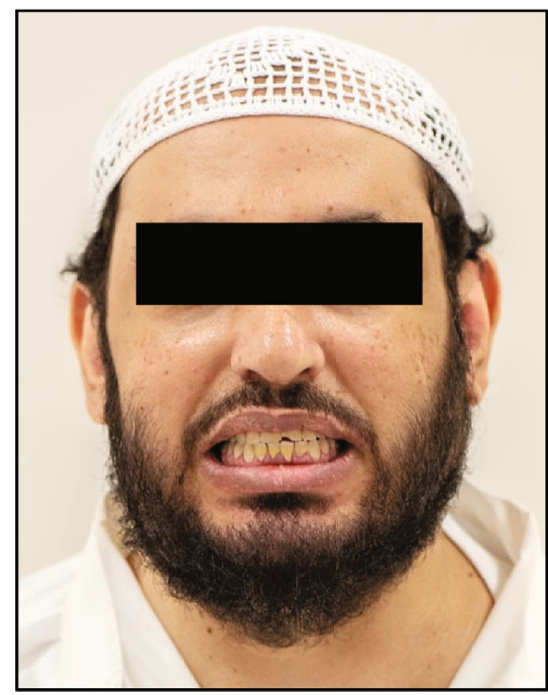

(e)

FIgURE 6: Postoperative clinical photograph taken 7 years postsurgery with facial gestures eliciting unrestrained action of different muscles of facial expression. (a) Unremarkable healing of the surgical wound without any scarring and the patient is seen smiling. (b) Frontal facial view showing symmetric appearance and the patient is seen puffing the cheeks. (c, d) Bilateral symmetric eyelid closure and opening. (e) The patient is seen grinning broadly.

knowledge about vascularity of the tumor and MRI shows the relationship of the tumor to surrounding vital structures in the head and neck regions [11].

The treatment of PA irrespective of their size, severity, or malignant potential is only by surgery. Based on our litera- ture review, giant PA involving the superficial lobe of parotid was managed either by SP or ECD [4, 10, 24, 26-29, 31, 32]. On the contrary, TP was reportedly done for tumors exhibiting malignant characteristics $[8,12,15,17,19,20,23,30]$ and those involving both the deep and superficial lobes $[9,13,14$, 


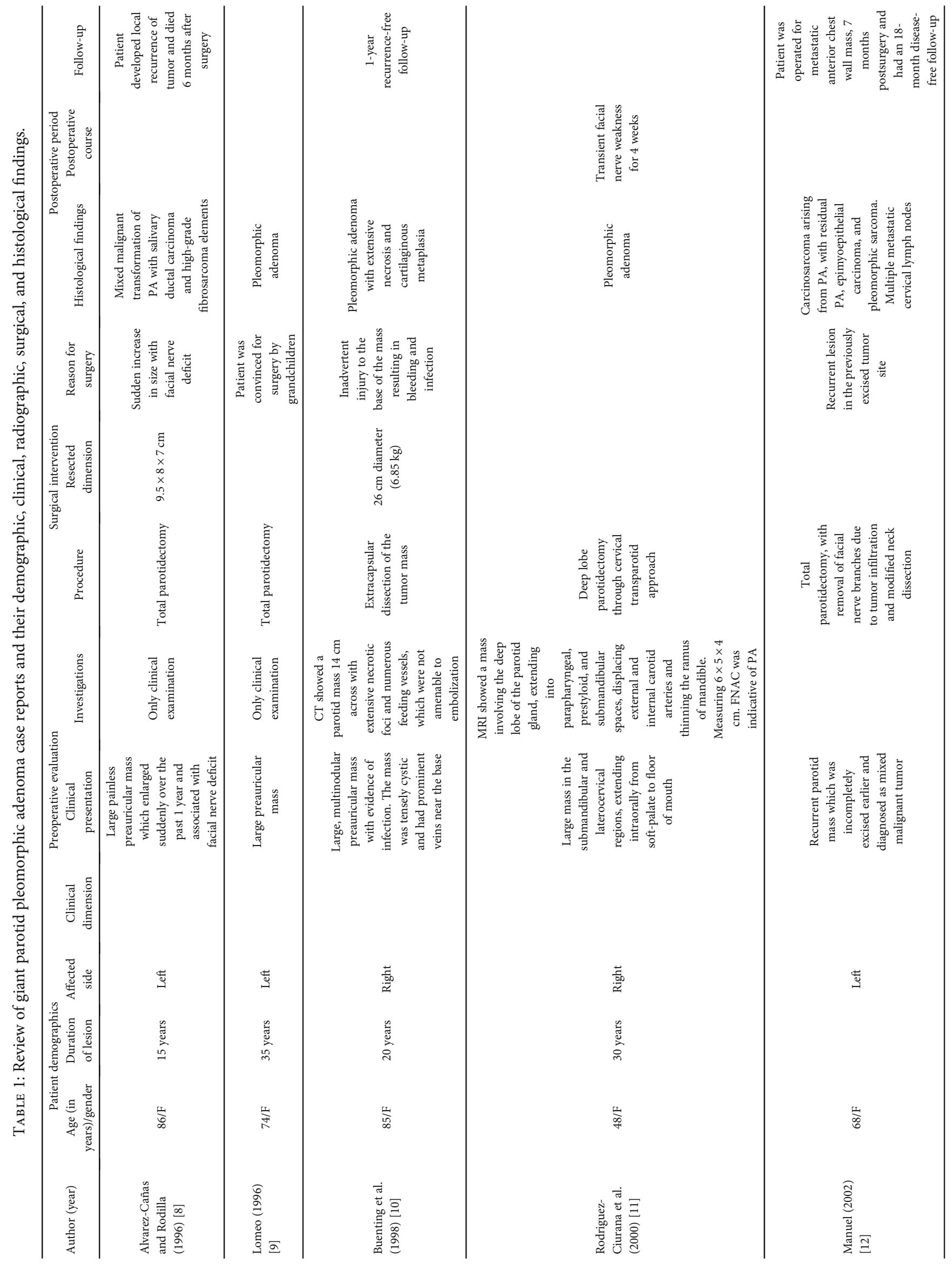




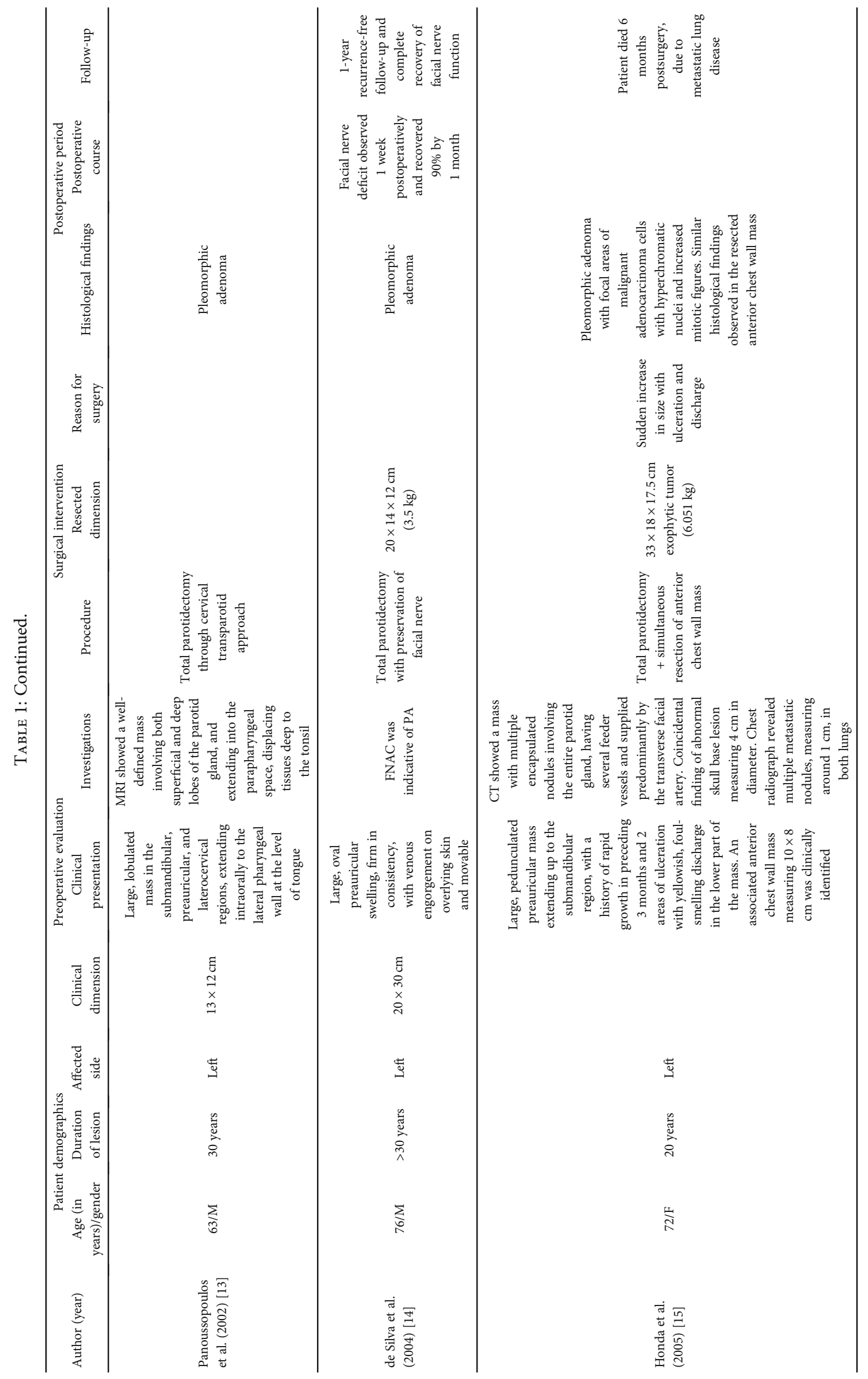




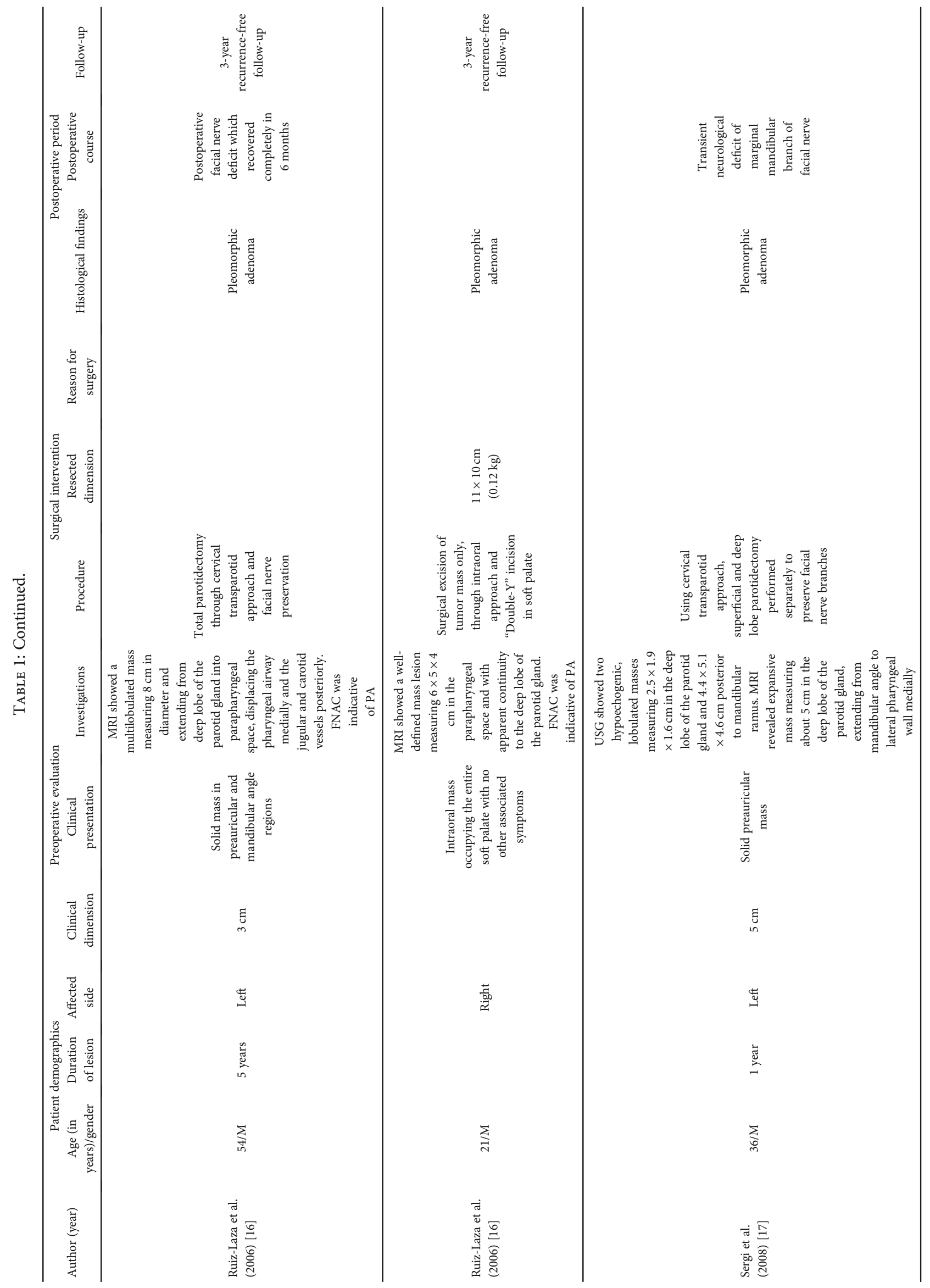



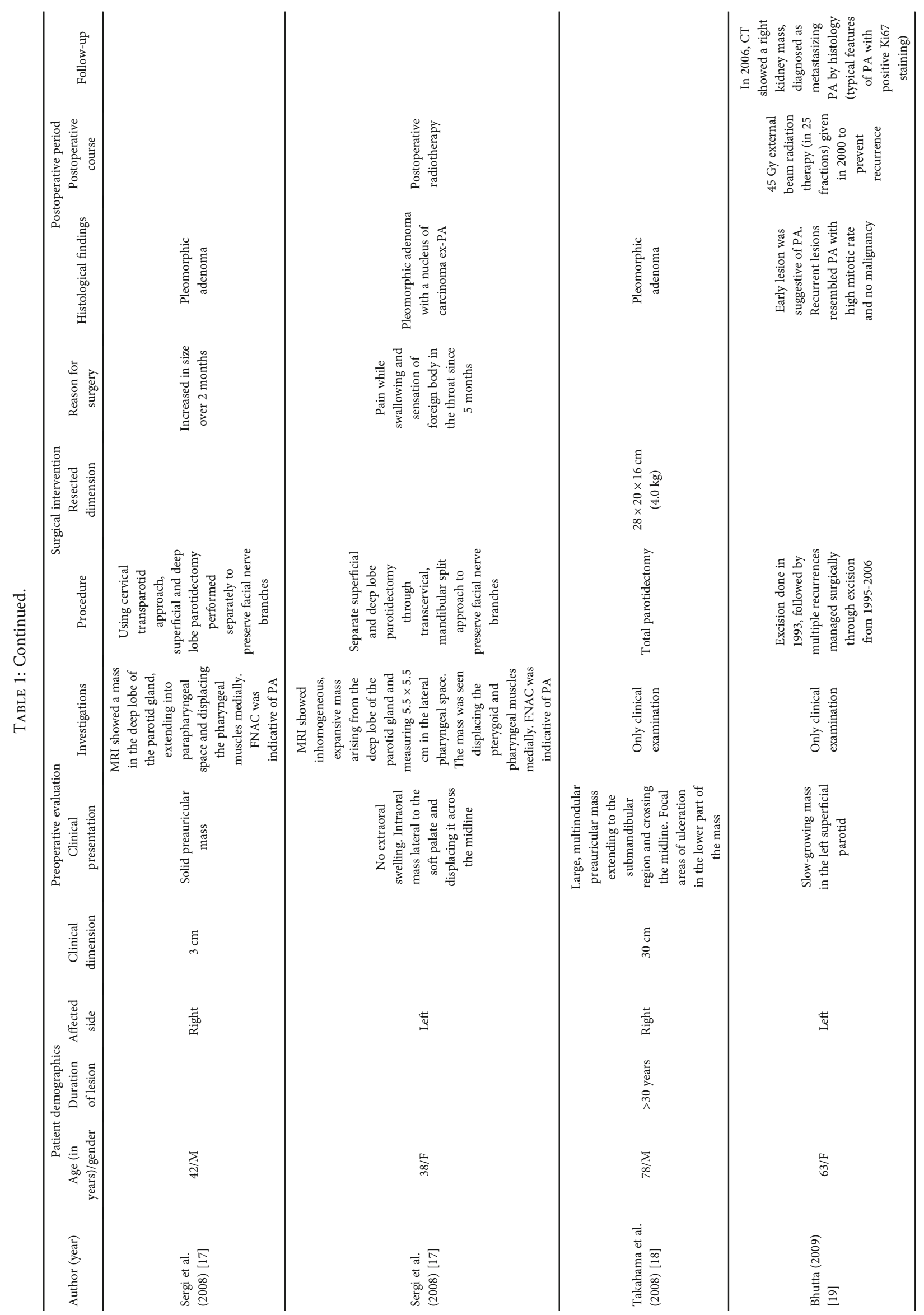


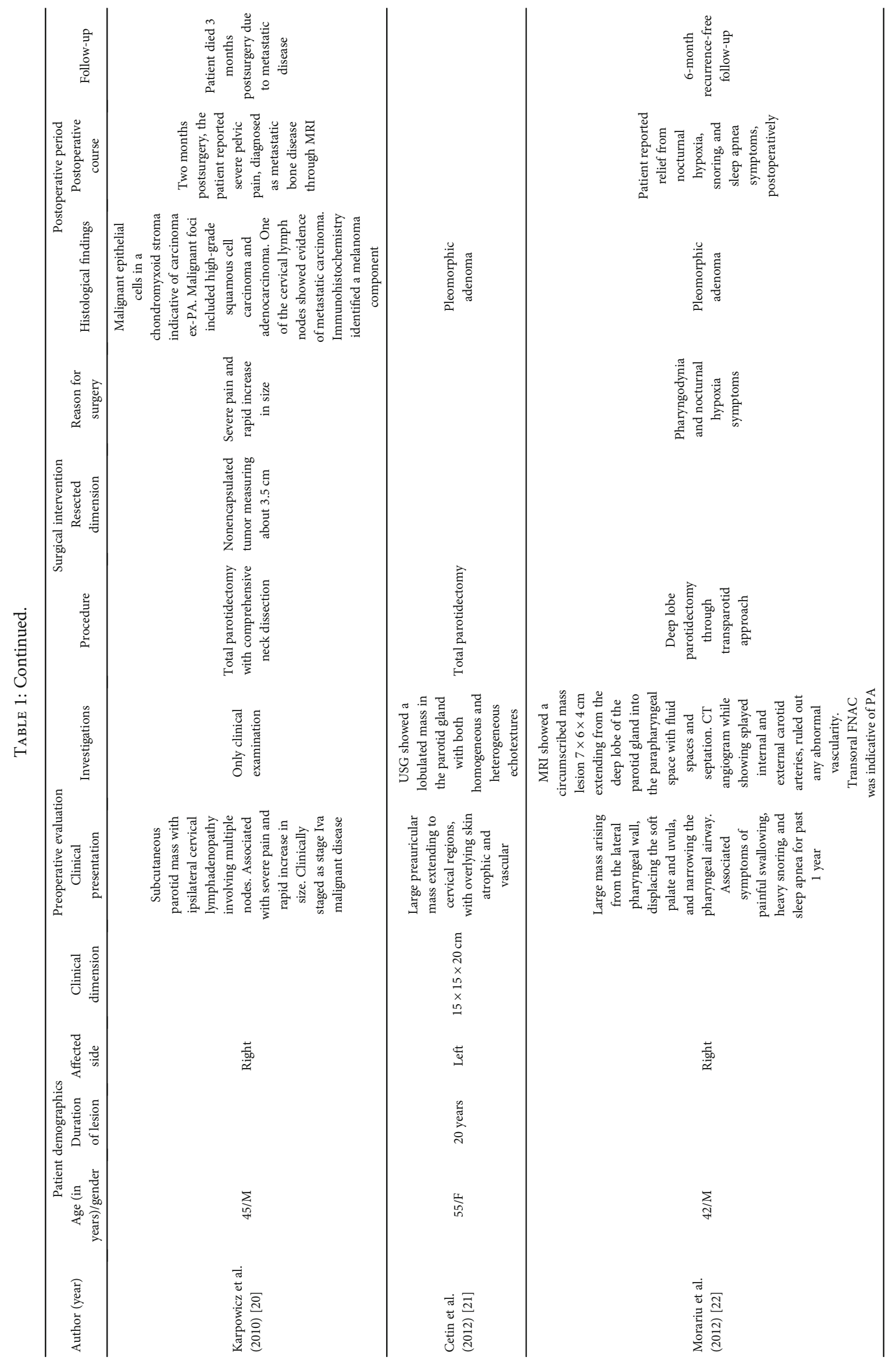




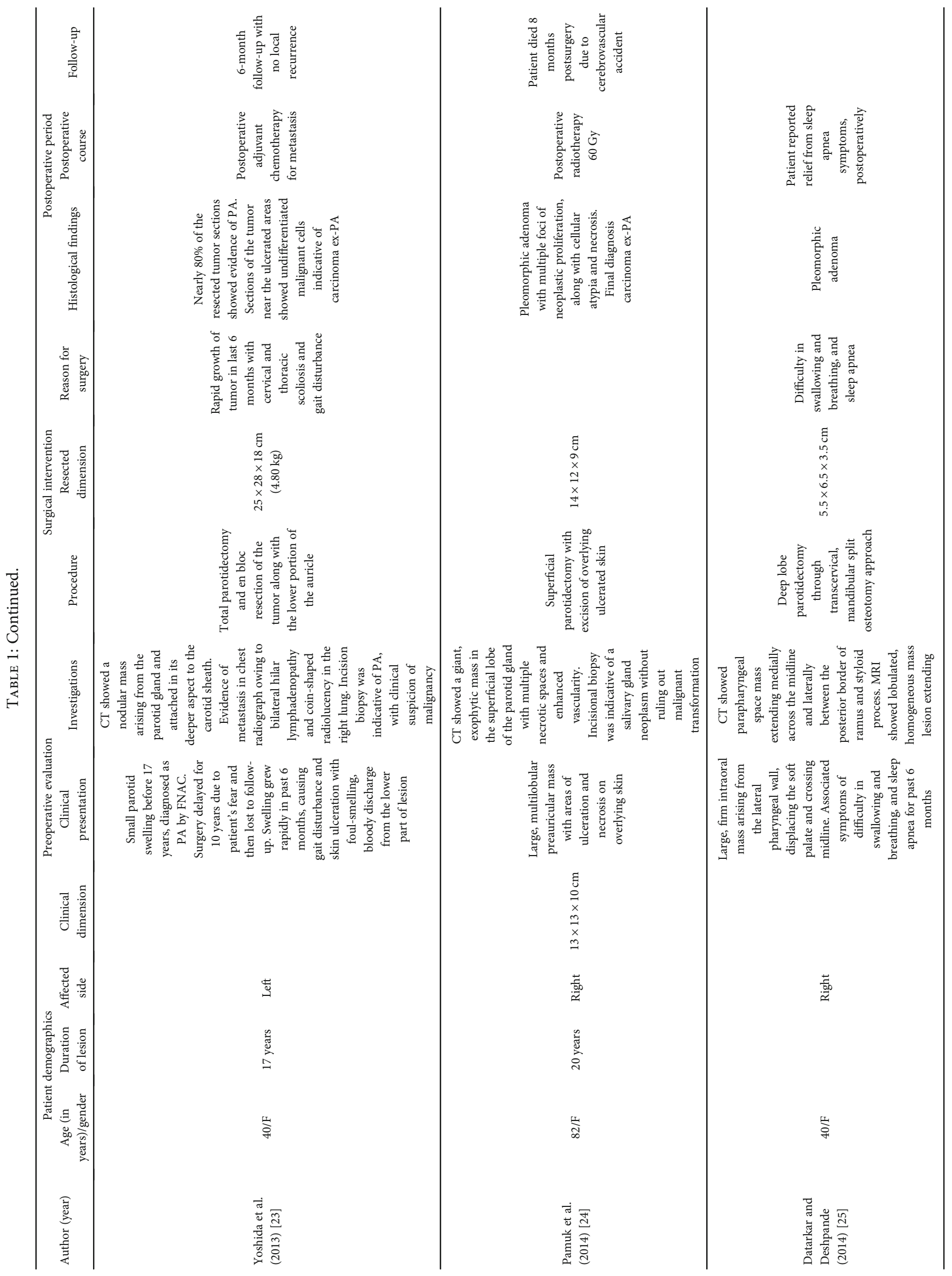




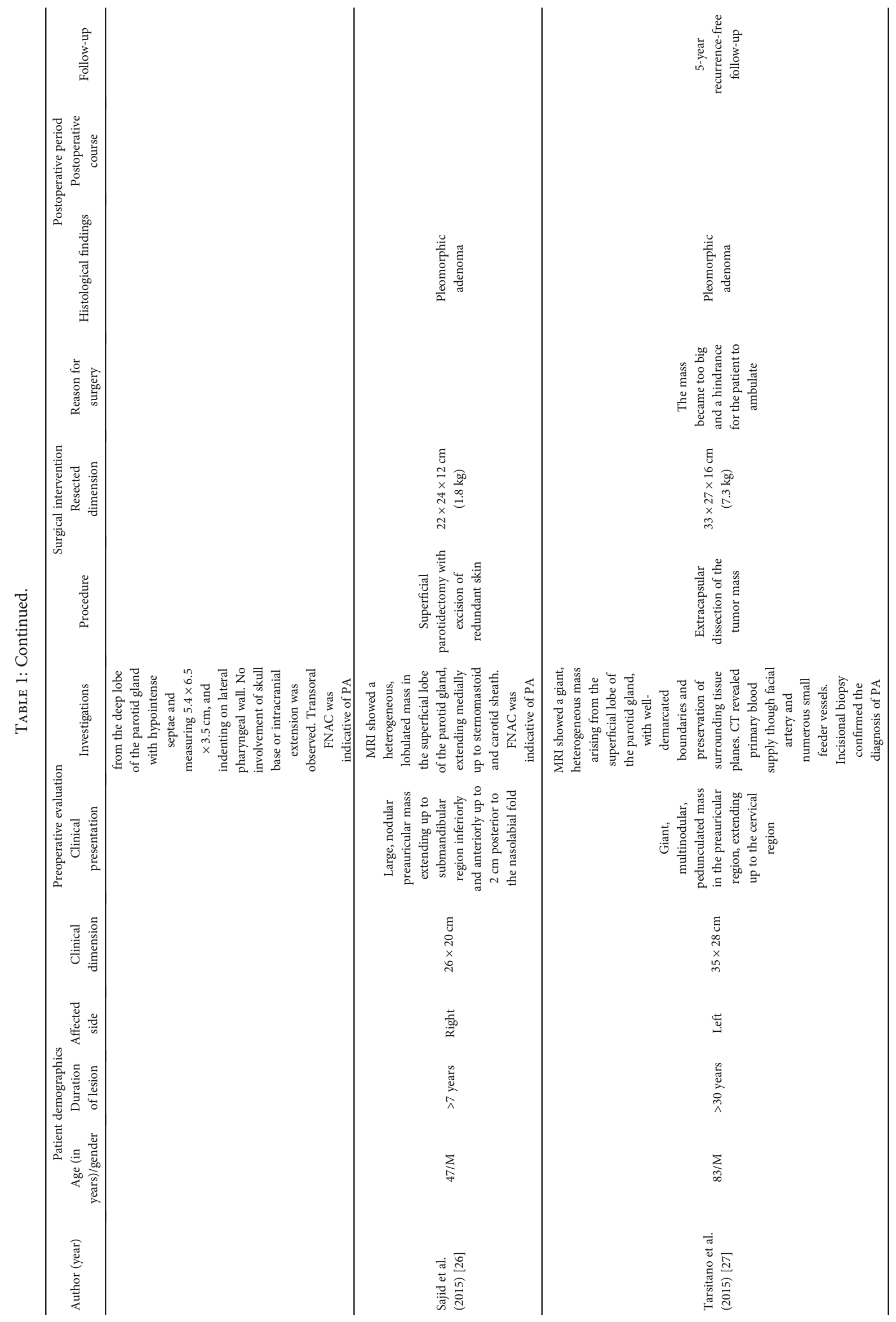




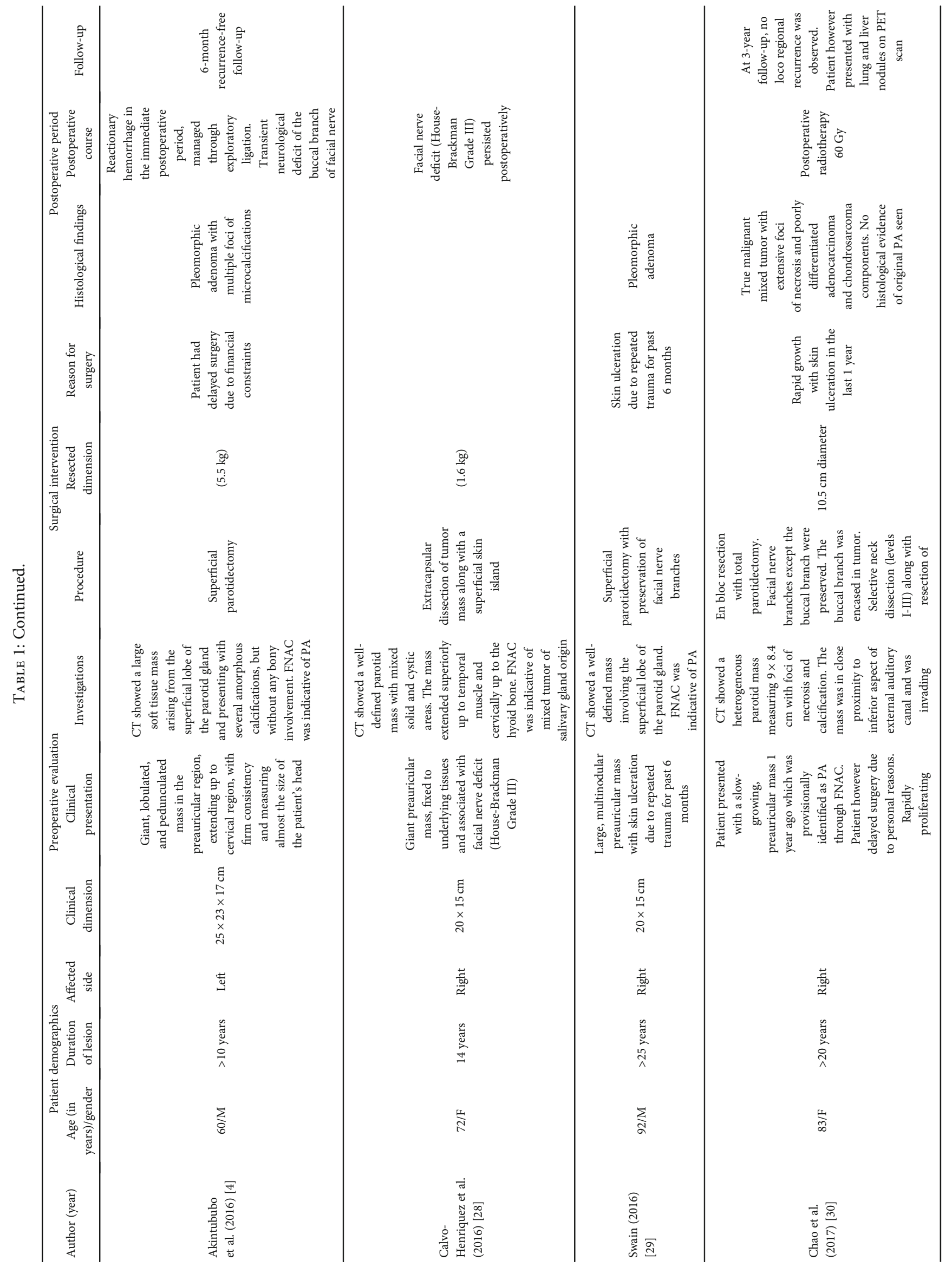




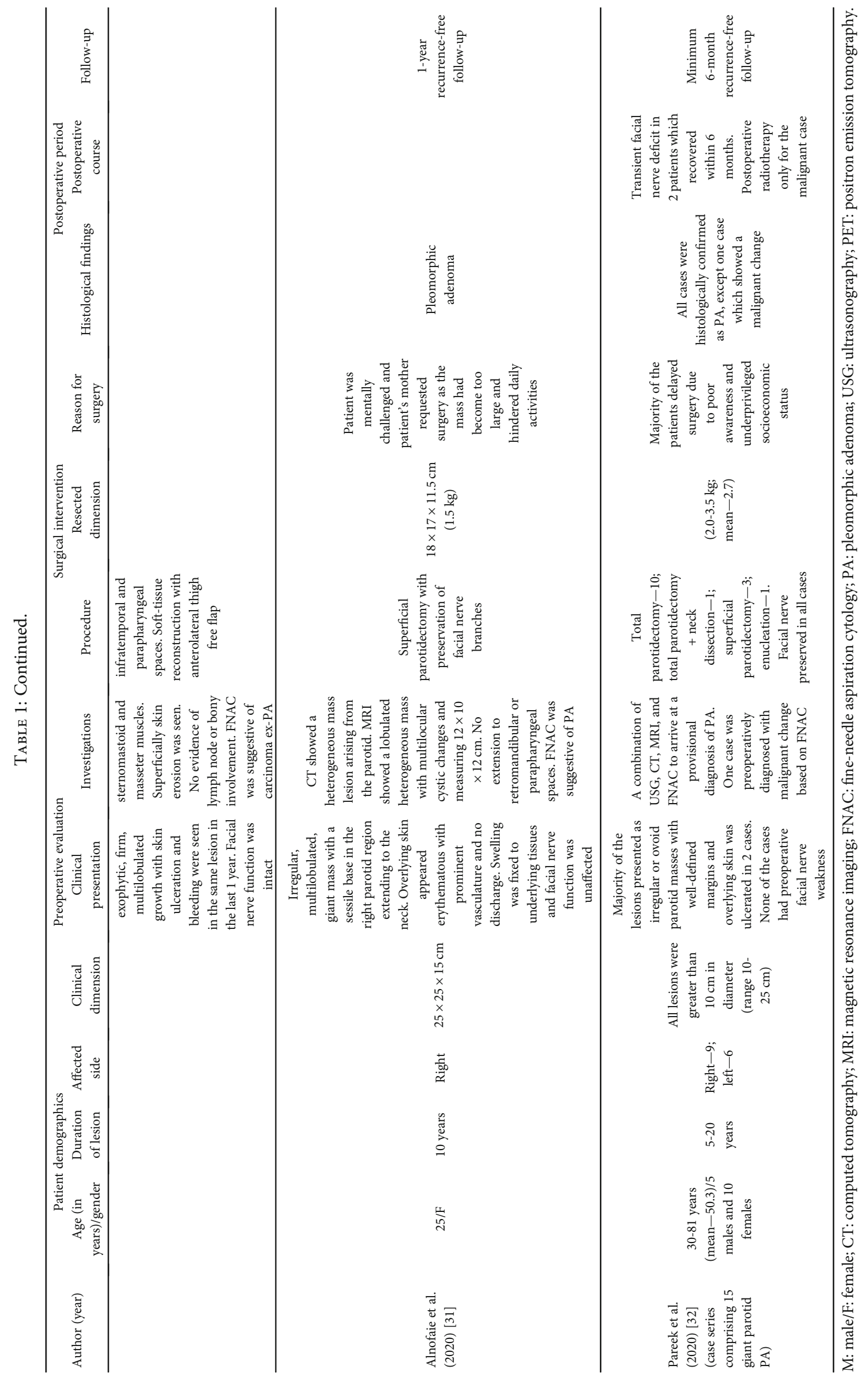


16-18, 21, 32] (Table 1). Since the present case was that of a PA involving superficial parotid only (Figures 1 and 2), the patient was surgically managed through ECD (Figure 3), avoiding any iatrogenic damage to the facial nerve (Figure 5). The surgical techniques for resecting PA have evolved over the last century and have essentially focused on preserving anatomic and functional integrity of the facial nerve and its branches $[1,7]$. While intracapsular removal was advocated, during the late $19^{\text {th }}$ and early $20^{\text {th }}$ centuries in an attempt to avoid facial nerve injury, the technique was abandoned due to its high risk of recurrence $(\geq 45 \%)$ $[1,7]$. This leads to the popularity of SP, associated with a very low recurrence rate $(\leq 2 \%)$, but still carrying a risk of facial nerve injury, Frey syndrome, and loss of facial contour [1]. In the last 2-3 decades, ECD has gained significance as a surgical technique which involves tumor resection along with the capsule and a thin rim of extracapsular normal tissue, without attempting to identify or dissect the facial nerve and its branches [1]. Based on literature reviews, Kato et al. [1] and Bonavolontà et al. [7] reported that ECD is safe, is economical, and reduces operating time and risk of morbidity. They further considered it as the treatment of choice for mobile, benign tumors involving the superficial lobe of the parotid gland [1, 7]. Moreover, traction due to gravity in giant PA causes the tumor mass to descend away from the facial nerve and its branches, thereby facilitating ease of dissection, as encountered in the present case [10].

Several surgical approaches to remove PA and other benign parotid gland tumors involving the deep lobe and extending to the lateral pharyngeal and parapharyngeal spaces have been reported [11]. These include cervical, cervical-transpharyngeal, infratemporal fossa, parotid-cervical, transoral, and transparotid approaches. In the present review, 9 cases of PA were reportedly involving the deep lobe of parotid and extending into pharyngeal spaces. The cervical-transparotid surgical approach was used for resection in 6 cases $[11,13,16,17,22]$, a transcervical, splitmandibulotomy approach was used in 2 cases [17, 25], and one case was managed through a transoral approach using "Double-Y" incision in soft palate [16] (Table 1). Postoperative complications following surgical resection of parotid PA are similar to that of other benign SGT $[1,4,7,23]$. These include hemorrhage, hematoma, seroma, fistula, hypoesthesia, scarring, Frey syndrome, transient facial nerve injury, and facial paralysis [7]. While these complications are reportedly associated with all surgical techniques mentioned before, ECD was associated with a significantly lower risk of hypoesthesia, Frey syndrome, and facial nerve injury and paralysis [7]. Nevertheless, when dealing with large parotid tumors with suspected malignancy, which mandate identification and dissection of facial nerve branches, no significant differences were observed in the outcomes and complications between antegrade and retrograde dissection techniques [30]. Postoperative complications reported in the current review include reactionary hemorrhage immediately after surgery [4] and transient facial nerve deficit which recovered in durations ranging from one month to 6 months $[4,11,14,16,17$, 32] (Table 1). Although there were no documented cases of permanent facial nerve injury, Calvo-Henriquez et al. [28] reported a case of preoperative "House-Brackman Grade III" facial nerve deficit, which persisted postoperatively.

In addition to the long-standing nature of the tumor and its large size, the currently reported case also presented with atypical histological findings. While the histological picture was predominantly coherent with that ofPA characterized by epithelial tissue dispersed in a chondroid and myxoid matrix [34], there was marked squamous metaplasia and keratin cyst formation (Figure 4). These findings are unusual in PA of the parotid gland and lead to a high index of suspicion towards a diagnosis of mucoepidermoid carcinoma or squamous cell carcinoma [35]. However, similar findings have been reported in the literature in PA arising in labial and palatal minor salivary glands, wherein malignant transformation is highly suspected and ranges from 2 to $23 \%[6,35]$. Squamous metaplasia of glandular cells is attributed to hypoxia within the growing tumor and combined with keratin cysts; they mimic cutaneous appendages and are termed as "cystic pleomorphic adenoma with extensive adnexa-like differentiation" [35]. It has further been emphasized that the above findings during the histological examination of suspected pleomorphic adenomas warrant meticulous histopathological evaluation to avoid surgical overtreatment [6, 35]. Analyzing the histological findings in the present review, only one case was associated with cartilaginous metaplasia [10], without any evidence of malignant change, similar to that observed in our case too. Nevertheless, malignant transformation was reported based on final histopathological examination in 10 cases, including 5 cases of carcinoma expleomorphic adenoma (CA-ExPA) [17, 20, 23, 24, 32], one case of adenocarcinoma along with PA [15], 3 cases of mixed malignant tumor arising in PA $[8,12,30]$, and one case of metastasizing PA with renal metastasis [19] (Table 1). Interestingly, one of the cases of CA-ExPA also had a malignant melanoma component, arising possibly from melanocytes within parotid tissue [20].

Malignant transformation of PA is reported at around $12 \%$ and comprises nearly $4 \%$ of all SGT [20]. According to the World Health Organization (WHO) classification of SGT, malignant tumors arising in PA were originally classified either into CA-ExPA or the relatively rare mixed malignant tumor with epithelial and mesenchymal components [8, 12]. While metastasizing PA, with metastatic lesions exhibiting histological characteristics of PA and no malignant change was originally considered a malignant variant, it has been relegated to the benign category as per the WHO classification (2017) of SGT [36]. Although rare, metastasizing PA is frequently associated with multiple recurrences of the primary tumor. Bhutta et al. [19] reported a case of metastatic renal PA, with multiple postsurgical recurrences of the parotid PA in over a decade. Based on a retrospective study of 221 patients with PA and 15 patients with CA-ExPA, Seok et al. [37] reported increasing age, regional lymph node involvement $(>5 \mathrm{~mm})$, and MRI apparent diffusion coefficient as the distinguishing clinical and radiographic features of CA-ExPA. In terms of histology, cellular atypia and stromal hyalinization have been regarded as the key delineating features of CA-ExPA from benign PA $[36,38]$. Based on immunohistochemistry (IHC), loss of expression of PLAG- 
1 (pleomorphic adenoma gene-1) protein and overexpression of Ki-67, p53, and HER-2 (human epidermal growth factor receptor-2) are considered suggestive of malignant transformation in otherwise benign PA $[38,39]$. At a molecular genetic level, malignant transformation of PA is further characterized by PLAG-1/HMGA-2 (High Mobility Group AT-Hook 2) fusion and rearrangement, p53 mutation, and HER-2 amplification [36, 38, 39].

In the present review, PA with malignant transformation was treated surgically, followed by radiotherapy for locoregional disease and chemotherapy for distant metastases. Within postoperative follow-up periods ranging from 6 months to 5 years, only one case of recurrent benign PA was reported in the review [19]. Among tumors with malignant change, locoregional recurrence was reported in one case of mixed malignant tumor [8], cervical lymph node metastasis was seen in 2 cases [12, 20], and distant metastasis to the anterior chest wall, lungs, liver, pelvis, and kidney was observed in 5 cases $[12,15,19,20,30]$. While there was no mortality associated with benign PA or its surgical treatment, 3 patients with malignant transformation died within 6 months after surgery as a result of metastatic disease $[8,15$, 20]. The longest recurrence-free follow-up for a giant parotid PA in the present review was 5 years [27], and this was still lesser than the 7-year recurrence-free follow-up observed in our case.

\section{Conclusion}

Giant parotid PA is rare, and to the best of our knowledge from indexed literature, this is one of the very few cases reported in this geographical region (Saudi Arabia). Apart from its rarity, the present case is being reported for its long-standing clinical presentation, successful surgical management through ECD without any postoperative complications or facial nerve deficit, atypical histological presentation, and long-term recurrence-free follow-up. In addition to complete tumor removal, ECD helped preserve the integrity of facial nerve branches. Interestingly, the present case of giant parotid PA was benign in spite of associated squamous metaplasia and keratin cyst formation identified histologically in the resected specimen. Nevertheless, this mandated a long-term follow-up of the patient. Therefore, based on this case report, clinicians must be aware of atypical histological presentation associated with parotid PA and plan suitable surgical management and follow-up, to achieve complete tumor removal and avoid morbidity. Lastly, attempts must be made to diagnose and manage PA at an early stage and before they reach gigantic proportions, through better public health care initiatives and creating awareness among all physicians and patients.

\section{Ethical Approval}

This case report was ethically approved by the ethical committee at the College of Dentistry Research Center, King Saud University, Riyadh, Saudi Arabia.

\section{Consent}

Informed consent was obtained from the patient.

\section{Conflicts of Interest}

The authors report no conflicts of interest with respect to the publication of this article.

\section{References}

[1] M. G. Kato, E. Erkul, S. A. Nguyen et al., "Extracapsular dissection vs superficial parotidectomy of benign parotid lesions," JAMA Otolaryngology- Head \& Neck Surgery, vol. 143, no. 11, pp. 1092-1097, 2017.

[2] A. Reinheimer, D. S. Vieira, M. M. Cordeiro, and E. R. Rivero, "Retrospective study of 124 cases of salivary gland tumors and literature review," Journal of Clinical and Experimental Dentistry, vol. 11, no. 11, pp. e1025-e1032, 2019.

[3] O. E. Ogle, "Salivary gland diseases," Dental Clinics of North America, vol. 64, no. 1, pp. 87-104, 2020.

[4] O. B. Akintububo, O. K. Ogundipe, Z. Y. Kaltungo, M. I. Guduf, U. H. Pindiga, and Y. M. Abdullahi, "Giant parotid pleomorphic adenoma in a Nigerian male," Nigerian Journal of Clinical Practice, vol. 19, no. 5, pp. 681-684, 2016.

[5] A. Rai, S. Sharma, P. Shrivastava, and M. Singh, "A huge pleomorphic adenoma of the submandibular salivary gland," $B M J$ Case Reports, vol. 2018, 2018.

[6] S. Sharma, M. Mehendiratta, N. Chaudhary, V. Gupta, M. Kohli, and A. Arora, "Squamous metaplasia in pleomorphic adenoma: a diagnostic and prognostic enigma," Journal of Pathology and Translational Medicine, vol. 52, no. 6, pp. 411-415, 2018.

[7] P. Bonavolontà, G. Dell'Aversana Orabona, F. Maglitto et al., "Postoperative complications after removal of pleomorphic adenoma from the parotid gland: a long-term follow up of 297 patients from 2002 to 2016 and a review of publications," The British Journal of Oral \& Maxillofacial Surgery, vol. 57, no. 10, pp. 998-1002, 2019.

[8] C. Alvarez-Cañas and I. G. Rodilla, "True malignant mixed tumor (carcinosarcoma) of the parotid gland," Oral Surgery, Oral Medicine, Oral Pathology, Oral Radiology, and Endodontology, vol. 81, no. 4, pp. 454-458, 1996.

[9] P. E. Lomeo, "Giant pleomorphic adenoma of the parotid," Ear, Nose, \& Throat Journal, vol. 75, p. 402, 1996.

[10] J. E. Buenting, T. L. Smith, and D. K. Holmes, "Giant pleomorphic adenoma of the parotid gland: case report and review of the literature," Ear, Nose, \& Throat Journal, vol. 77, pp. 634, 637-638, 640, 1998.

[11] J. Rodriguez-Ciurana, C. Rodado, M. Saez, and C. Bassas, "Giant parotid pleomorphic adenoma involving the parapharyngeal space: report of a case," Journal of Oral and Maxillofacial Surgery : official journal of the American Association of Oral and Maxillofacial Surgeons, vol. 58, no. 10, pp. 11841187, 2000.

[12] S. Manuel, A. Mathews, K. Chandramohan, and M. Pandey, "Carcinosarcoma of the parotid gland with epithelial-myoepithelial carcinoma and pleomorphic sarcoma components," British Journal of Oral and Maxillofacial Surgery, vol. 40, no. 6, pp. 480-483, 2002.

[13] D. Panoussopoulos, J. Yotakis, B. Pararas, G. Theodoropoulos, and K. Papadimitriou, "Giant pleomorphic adenoma of the 
parotid gland involving the parapharyngeal space treated by a totally extraoral transparotid approach," Journal of Surgical Oncology, vol. 81, no. 3, pp. 155-157, 2002.

[14] M. N. de Silva, K. M. S. Kosgoda, W. M. Tilakaratne, and P. Murugadas, "A case of giant pleomorphic adenoma of the parotid gland," Oral Oncology Extra, vol. 40, no. 3, pp. 43-45, 2004.

[15] T. Honda, Y. Yamamoto, T. Isago, H. Nakazawa, M. Nozaki, and T. Hirayama, "Giant pleomorphic adenoma of the parotid gland with malignant transformation," Annals of Plastic Surgery, vol. 55, no. 5, pp. 524-527, 2005.

[16] L. Ruiz-Laza, P. Infante-Cossio, A. Garcia-Perla, J.M. Hernandez-Guisado, and J.-L. Gutierrez-Perez, "Giant pleomorphic adenoma in the parapharyngeal space: report of 2 cases," Journal of Oral and Maxillofacial Surgery, vol. 64, no. 3, pp. 519-523, 2006.

[17] B. Sergi, A. Limongelli, E. Scarano, A. R. Fetoni, and G. Paludetti, "Giant deep lobe parotid gland pleomorphic adenoma involving the parapharyngeal space. Report of three cases and review of the diagnostic and therapeutic approaches," Acta Otorhinolaryngologica Italica : Organo Ufficiale della Societa Italiana di Otorinolaringologia e Chirurgia Cervico-Facciale, vol. 28, no. 5, pp. 261-265, 2008.

[18] A. Takahama Jr., D. E. da Cruz Perez, J. Magrin, O. P. de Almeida, and L. P. Kowalski, "Giant pleomorphic adenoma of the parotid gland," Medicina Oral, Patologia Oral y Cirugia Bucal, vol. 13, pp. E58-E60, 2008.

[19] M. F. Bhutta, L. Dunk, A. J. Molyneux, and A. Tewary, "Parotid pleomorphic adenoma with solitary renal metastasis," British Journal of Oral and Maxillofacial Surgery, vol. 48, no. 1, pp. 61-63, 2010.

[20] M. K. Karpowicz, B. Shalmon, K. H. Molberg, and A. K. ElNaggar, "Melanoma in a carcinoma ex pleomorphic adenoma of the parotid gland: a case report and putative histogenesis," Human Pathology, vol. 42, no. 9, pp. 1355-1358, 2011.

[21] M. A. Cetin, A. Ikinciogullari, G. Saygi, H. G. Hatipoglu, S. Koseoglu, and H. Dere, "Giant pleomorphic adenoma of the parotid gland," The Turkish Journal of Ear Nose and Throat, vol. 22, no. 2, pp. 116-118, 2012.

[22] I. Morariu, A. Dias, and A. Curran, "Giant parotid pleomorphic adenoma in parapharyngeal space causing severe obstructive sleep apnoea," Irish Medical Journal, vol. 105, no. 6, pp. 184-185, 2012.

[23] N. Yoshida, M. Hara, H. Kanazawa, and Y. Iino, "Large carcinoma ex pleomorphic adenoma of the parotid gland: a case report and review of literature," Journal of Oral and Maxillofacial Surgery, vol. 71, no. 12, pp. 2196.e1-2196.e6, 2013.

[24] A. E. Pamuk, C. Cabbarzade, H. Uner, R. Ö. Günaydın, and K. Kosemehmetoglu, "A neglected giant parotid gland mass: excision and reconstruction with facial nerve preservation," Otolaryngologia Polska, vol. 68, no. 6, pp. 333-337, 2014.

[25] A. N. Datarkar and A. Deshpande, "Giant parapharyngeal space pleomorphic adenoma of the deep lobe of parotid presenting as obstructive sleep apnoea: a case report \& review of the diagnostic and therapeutic approaches," Journal of Maxillofacial and Oral Surgery, vol. 14, no. 3, pp. 532-537, 2015.

[26] M. Sajid, S. Rehman, and J. Misbah, "Giant pleomorphic adenoma of the parotid gland," Journal of the College of Physicians and Surgeons-Pakistan, vol. 25, Supplement 2, pp. S110-S111, 2015.

[27] A. Tarsitano, A. Pizzigallo, F. Giorgini, and C. Marchetti, "Giant pleomorphic adenoma of the parotid gland: an unusual case presentation and literature review," Acta Otorhinolaryngologica Italica: Organo Ufficiale della Societa Italiana di Otorinolaringologia e Chirurgia Cervico-Facciale, vol. 35, no. 4, pp. 293-296, 2015.

[28] C. E. Calvo-Henriquez, A. Telmo-Mella, C. Dios-Loureiro, and C. Martin-Martin, "Giant pleomorphic adenoma of the parotid gland," Acta Otorrinolaringologica Espanola, vol. 67, no. 6, pp. e40-e41, 2016.

[29] S. K. Swain, M. C. Sahu, and S. Mishra, "An ulcerated giant pleomorphic adenoma of the parotid gland - a case report," Egyptian Journal of Ear, Nose, Throat and Allied Sciences, vol. 17, no. 2, pp. 111-114, 2016.

[30] T. N. Chao, C. H. Rassekh, S. B. Cannady, and V. A. LiVolsi, "Giant parotid carcinosarcoma arising in a pleomorphic adenoma: facial nerve preservation by retrograde dissection," OTO open, vol. 1, no. 3, p. 2473974X1771941, 2017.

[31] H. Alnofaie, T. Alshammeri, Y. Alturkistany, A. Aljabab, and A. Alweteid, "Giant pleomorphic adenoma of parotid gland in Saudi Arabia: a rare case report," SN Comprehensive Clinical Medicine, vol. 2, no. 8, pp. 1258-1263, 2020.

[32] Y. K. Pareek, D. Gupta, Y. Aseri, D. S. Rawat, B. K. Singh, and P. C. Verma, "Giant pleomorphic adenomas of parotid gland: a case series," Indian Journal of Otolaryngology and Head \& Neck Surgery, 2020.

[33] S. Jousse-Joulin, "Chapter 17 - salivary glands," in Essential Applications of Musculoskeletal Ultrasound in Rheumatology, R. J. Wakefield and M. A. D'Agostino, Eds., pp. 199-206, W.B. Saunders, Philadelphia, 2010.

[34] D. K. Das and J. T. Anim, "Pleomorphic adenoma of salivary gland: to what extent does fine needle aspiration cytology reflect histopathological features?," Cytopathology : official journal of the British Society for Clinical Cytology, vol. 16, no. 2, pp. 65-70, 2005.

[35] M. C. V. Goulart, P. Freitas-Faria, G. R. Goulart et al., "Pleomorphic adenoma with extensive squamous metaplasia and keratin cyst formations in minor salivary gland: a case report," Journal of Applied Oral Science : Revista FOB, vol. 19, no. 2, pp. 182-188, 2011.

[36] R. R. Seethala and G. Stenman, "Update from the 4th edition of the World Health Organization Classification of Head and Neck Tumours: tumors of the salivary gland," Head and Neck Pathology, vol. 11, no. 1, pp. 55-67, 2017.

[37] J. Seok, S. J. Hyun, W. J. Jeong, S. H. Ahn, H. Kim, and Y. H. Jung, "The difference in the clinical features between carcinoma ex pleomorphic adenoma and pleomorphic adenoma," Ear, Nose, \& Throat Journal, vol. 98, no. 8, pp. 504-509, 2019.

[38] S. Di Palma, "Carcinoma ex pleomorphic adenoma, with particular emphasis on early lesions," Head and Neck Pathology, vol. 7, no. S1, pp. 68-76, 2013.

[39] M. Asahina, T. Saito, T. Hayashi, Y. Fukumura, K. Mitani, and T. Yao, "Clinicopathological effect of plag1 fusion genes in pleomorphic adenoma and carcinoma ex pleomorphic adenoma with special emphasis on histological features," Histopathology, vol. 74, no. 3, pp. 514-525, 2019. 